\title{
器 \\ Estudo de prevalência de parasitos zoonóticos em amostras de fezes de cães em vias públicas de quatro bairros do município de Pelotas-RS
}

\author{
[Study of the prevalence of zoonotic parasites in faecal samples of dogs on public roads in four \\ neighborhoods of the city of Pelotas, $R S]$
}

\section{"Artigo Científico/Scientific Article"}

\author{
Bruno Cabral Chagas ${ }^{1 *}$, Jaqueline Freitas Motta ${ }^{2}$, Alexsander Ferraz ${ }^{3}$, Sergio Silva Silva ${ }^{3}$, \\ Catia Cilene Santos Mello ${ }^{4}$, Lariss Dall’Agnol Silva ${ }^{5}$, Leandro Quintana Nizoli ${ }^{3}$
}

\author{
${ }^{1}$ Departamentoํㅡㄹ \\ ${ }^{2}$ Departamento de Biotecnologia, Universidade Federal de Pelotas, Pelotas-RS, Brasil. \\ ${ }^{3}$ Departamento de Veterinária Preventiva, Universidade Federal de Pelotas, Pelotas-RS, Brasil. \\ ${ }^{4}$ Departamento de Biologia, Universidade Federal de Pelotas, Pelotas-RS, Brasil. \\ ${ }^{5}$ Departamento de Terapia Ocupacional, Universidade Federal de Pelotas, Pelotas-RS, Brasil. \\ *Autor para correspondência/Corresponding author: E-mail: brunocabral.chagas@gmail.com
}

\begin{abstract}
Resumo
As parasitoses zoonóticas transmitidas por animais de companhia possuem um caráter de agressão nos próprios animais e em seres humanos em alguns casos e ainda tem pouca divulgação junto à comunidade. $\mathrm{O}$ objetivo deste trabalho foi estimar a prevalência de parasitos zoonóticos em quatro bairros do município de Pelotas-RS. Foram realizadas coletas de amostras de fezes de caninos em vias públicas referente aos bairros Bom Jesus, Simões Lopes, Navegantes e Barro Duro, processadas pela técnica de Willis-Mollay (1921). Os parasitos zoonóticos encontrados foram o Ancylostoma spp. no intervalo de prevalência de 50\% a 76,39\%, Toxocara spp. entre 7,38\% a 53\%, Trichuris vulpis entre $18,06 \%$ a $25,26 \%$ e outros dois em menores prevalências como Toxascaris $(0,48 \%)$ e Cestódeos $(1,22 \%)$. Estes dados demonstraram a importância e o cuidado que se deve ter com animais de estimação preferencialmente os domiciliados e animais comunitários, bem como a importância que estes possuem na disseminação e continuidade de doenças zoonóticas as quais a população está frequentemente exposta.
\end{abstract}

Palavras-chave: saúde pública; diagnóstico coprológico; animais de estimação; doenças parasitárias.

\begin{abstract}
Zoonotic parasites transmitted by companion animals have a character of aggression in the animals themselves and in humans in some cases and still have little divulgation within the community. The objective of this work was to estimate the prevalence of zoonotic parasites in four neighborhoods of the city of Pelotas, RS. Samples of canine feces were taken from public streets in Bom Jesus, Simões Lopes, Navegantes, and Barro Duro neighborhoods, and processed using the Willis-Mollay technique (1921). The zoonotic parasites found were Ancylostoma spp. in the prevalence range of 50\% to 76.39\%, Toxocara spp. between $7.38 \%$ and 53\%, Trichuris vulpis between $18.06 \%$ and $25.26 \%$, and other two in lower prevalences, namely Toxascaris $(0.48 \%)$ and Cestodes $(1.22 \%)$. These data demonstrated the importance and care that should be given to pets, preferably the domiciled and communal animals, as well as their importance in the dissemination and continuity of zoonotic diseases to which the population is frequently exposed.
\end{abstract}

Keywords: public health; coprological diagnosis; pets; parasitic diseases.

\section{Introdução}

Doenças parasitárias de cunho zoonótico são endêmicas em grande parte do mundo e possuem fatores como condições climáticas favoráveis, falta de hábitos de higiene da população (Paiva et al., sua transmissão continuada em virtude de diversos 2014), descarte incorreto de vísceras de animais e 
manejo inadequado com dejetos dos mesmos (Pastore et al., 2003). Os cães são portadores de diversos parasitos internos como, Toxocara spp., Ancylostoma spp., Trichuris vulpis (Anaya et al., 2015), Dipylidium caninum, Echinococcus granulosus e Toxascaris sp. (Dantas-Torres e Otranto, 2014). Estes parasitos possuem ciclos evolutivos aonde o adulto disposto no sistema intestinal do cão, libera formas evolutivas nas fezes e estas, enquanto infectantes, poderão ser ingeridos por seres humanos e penetrar ativamente na pele na forma larval no caso do parasito Ancylostoma (Franke et al., 2011).

Algumas das parasitoses zoonóticas oriundas de cães nos seres humanos denominam-se como larva migrans visceral, larva migrans cutânea e tricuríase (Monteiro et al., 2016). Os sinais quando presentes, geralmente são inespecíficos variando do órgão e ou tecido que os parasitos ocasionalmente se estabeleçam, mas em geral, existem relatos de úlceras na mucosa intestinal, diarreia, melena (Ngui et al., 2014), alterações na função do sistema nervoso e hepático (Ma et al., 2018; Park et al., 2018), lesões pruriginosas de rápida evolução, eosinofilia (Soares et al., 2018) e no caso da larva migrans cutânea mais especificamente, lesões subcutânea com intenso prurido comumente nas extremidades das mãos e pés (González et al., 2015). A tricuríase causada pelo parasito Trichuris vulpis, já foi anteriormente incluída no complexo das larvas migrans, mas desde a década de 80 não há relatos que insiram esse parasito junto as larvas migrans, diferente da mantença de sua classificação como parasito zoonótico que vem se estabelecendo (Traversa e Donato, 2011) e não pode ser descartado frente aos quadros clínicos relatados a partir de sua ação como diarreia, desconforto abdominal e epistaxe (Márquez-Navarro et al., 2012).

A população estando cada vez mais próxima de cães como pets (Peacock et al., 2012) e convivendo com cães errantes e semi-domiciliados, está potencialmente expondo o animal e se expondo a um ambiente extremamente infestado por parasitos zoonóticos (Becker et al., 2012; Brilhante et al., 2013), o que demanda cuidados e reitera a importância da análise da presença destes junto ao ambiente.

A partir do exposto o objetivo deste trabalho foi investigar a prevalência dos parasitos zoonóticos dos gêneros Ancylostoma, Toxocara e Trichuris presentes em fezes de cães dispersas em vias públicas de quatro bairros do município de Pelotas-RS, Brasil.

\section{Material e Métodos}

A pesquisa foi realizada no município de Pelotas, situado a $31^{\circ} 46^{\prime} 19^{\prime}$ ' S $52^{\circ} 20^{\prime \prime} 34 \mathrm{O}$, região sul do estado do Rio Grande do Sul com predominância de clima subtropical. No período de 01 de maio de 2016 a 28 de outubro de 2016 foram realizadas coletas de fezes de cães em quatro bairros do município, sendo eles Barro Duro (zona leste), Simões Lopes (zona oeste), bairro Navegantes (zona leste) e Bom Jesus (zona leste).

As amostras de fezes foram selecionadas aleatoriamente, independente do seu estado de conservação, sendo recolhidas somente as que estivessem em via pública sem um horário fixo nos dias de coleta. As amostras pós coleta foram acondicionadas em recipientes plásticos identificados e mantidas sob refrigeração até seu processamento, o qual ocorreu em até quatro horas no Laboratório de doenças parasitárias (LADOPAR) da Universidade Federal de Pelotas.

O processamento das amostras de fezes foi realizado pela técnica de flutuação de WillisMollay (1921) modificada, com uso de solução hipersaturada $(1230 \mathrm{~g} / \mathrm{L})$ como análise qualitativa da presença de ovos de parasitos zoonóticos Ancylostoma spp., Toxocara spp. e Trichuris vulpis e parasitos potencialmente zoonóticos como Toxascaris e cestódeos os quais foram expressos em positivo e negativo. A aferição da presença ou ausência de ovos de parasitos zoonóticos foi realizada através do uso de microscópio binocular Olympus CX $21{ }^{\circledR}$ através de objetivas com planificações de $4 \mathrm{X}$ e $10 \mathrm{X}$.

Os dados obtidos foram acondicionados em planilhas eletrônicas e posteriormente analisados pelo cálculo de prevalência (O'Lorcain, 1994) pelo programa Statistica 6.0.

\section{Resultados e Discussão}

Do total de 410 amostras coletadas, foi encontrado uma prevalência elevada de parasitos do gênero Ancylostoma (63,81\%) (Tabela 1) causador da larva migrans cutânea em todos bairros pesquisados. A grande transmissão desses parasitos, pode ser vista também em várias regiões do globo, pela extensa capacidade deste em perpetuar-se em áreas com condições favoráveis de temperatura e umidade, sendo que na Malásia no sudeste asiático, Mahdy et al. (2012) diagnosticou a presença do gênero Ancylostoma em cães rurais 
$(71,4 \%)$, cães errantes urbanos $(48 \%)$ e cães de abrigo $(28,7 \%)$.

A prevalência de contaminação por Toxocara alcançou $12,51 \%$, assemelhando-se ao relatado na região sul do Brasil por Lopes et al. (2014) que diagnosticou uma diferença entre os valores de $39,16 \%$ de Ancylostoma em detrimento a contaminação por Toxocara que alcançou o patamar de $32,51 \%$. Essa diferença também é descrita em estudos realizados no Brasil central por Mota et al. (2014) que relataram a presença de 11,76\% de ovos de Ancylostoma e 3,92\% de ovos de Toxocara em amostras de cães provenientes do centro de controle de zoonoses de Ituitaba-MG. Também foi registrada uma semelhança na região norte do país por Labruna et al. (2006) com valores de $73,7 \%$ e $18,9 \%$ de prevalência de Ancylostoma spp. e Toxocara spp., respectivamente.

Tabela 1. Prevalência de contaminação dos parasitos zoonóticos encontrados em vias públicas na cidade de Pelotas-RS entre maio a outubro de 2016.

\begin{tabular}{lllllll}
\hline Parasitos & \multicolumn{2}{c}{ Ancylostoma } & \multicolumn{2}{c}{ Toxocara } & \multicolumn{2}{c}{ Trichuris vulpis } \\
\hline \multicolumn{1}{c}{ Bairros } & $\begin{array}{c}\text { Prevalência de } \\
\text { contaminação } \\
(\%)\end{array}$ & $\begin{array}{c}\mathbf{N}^{\mathbf{0}} \text { de } \\
\text { amostras } \\
\text { positivas }\end{array}$ & $\begin{array}{c}\text { Prevalência de } \\
\text { contaminação }\end{array}$ & $\begin{array}{c}\mathbf{N}^{\mathbf{0}} \text { de } \\
\text { amostras } \\
\text { positivas }\end{array}$ & $\begin{array}{c}\text { Prevalência de } \\
\text { contaminação } \\
(\%)\end{array}$ & $\begin{array}{c}\mathbf{N}^{\mathbf{0}} \text { de } \\
\text { amostras } \\
\text { positivas }\end{array}$ \\
\hline Simões Lopes & $76,39 \%$ & 55 & $15,28 \%$ & 11 & $18,06 \%$ & 13 \\
Barro Duro & $73,77 \%$ & 90 & $7,38 \%$ & 9 & $21,31 \%$ & 26 \\
Bom Jesus & $50,53 \%$ & 48 & $15,79 \%$ & 15 & $25,26 \%$ & 24 \\
Navegantes & $54,55 \%$ & 66 & $11,57 \%$ & 14 & $18,18 \%$ & 22 \\
\hline Média & $\mathbf{6 3 , 8 1}$ & & $\mathbf{1 2 , 5 1}$ & & $\mathbf{2 0 , 7 0}$ & \\
\hline
\end{tabular}

A menor contaminação de fezes dispersas no ambiente, por ovos de Toxocara spp. $(12,51 \%)$ encontrados neste trabalho, corroboram com Jenkins et al. (2014) que relataram a prevalência de $21,2 \%$ de Toxocara spp. em relação a 40,2 \% de contaminação por Trichuris vulpis, em 1425 cães da Austrália, já Johnson et al. (2015), diagnosticaram uma presença de $5,8 \%$ de prevalência de Toxocara canis quando em comparação a $46,8 \%$ de prevalência para outros nematódeos de cães domiciliados e peridomiciliados da região de Greatter Acra em Gana na região ocidental do contintente africano. A inferência dessa questão condiz com a forma de eliminação de parasitos, visto que ovos de Ancylostoma spp. são liberados praticamente em todas as fases de vida do animal e ovos de Toxocara spp. são eliminados primordialmente por filhotes enquanto imunodeprimidos por estes albergarem as formas adultas do parasito (Thomas e Jyathulakan, 2012).

O parasito Trichuris vulpis foi encontrado sob a média de prevalência de $20,70 \%$, resultado que vai de encontro com os dados expressos por Leite et al. (2007), o qual averiguou em levantamento epidemiológico realizado em um período de cinco anos na cidade de Curitiba-PR a partir de amostras de fezes caninas, uma prevalência de $10,35 \%$ de contaminação por ovos de Trichuris em contrapartida aos $18,6 \%$ de amostras contaminadas por ovos de Ancylostoma spp. Savilla et al. (2011) em teste realizado no estado da Virginia no sudeste dos Estados Unidos da América, revelou valores semelhantes ao diagnosticados neste trabalho, sendo A. caninum $23,4 \%$ o parasito de maior prevalência seguido de T. vulpis com $7,8 \%$ e $T$. canis com $6,9 \%$. Outros parasitos encontrados figuraram em contaminações menores como cestódeos $1,22 \%$, sendo que o bairro Barro Duro foi o que demonstrou maior contaminação com $2,45 \%$ seguido do bairro Simões Lopes com 1,64\% e as outras localidades não apresentaram infestação por cestódeos. $\mathrm{O}$ parasito Toxascaris spp. foi diagnosticado em $0,48 \%$ do total de amostras e somente encontrado na localidade do Simões Lopes em 2,78\%.

Os graves prejuízos à saúde pública estimulados pelos parasitos zoonóticos Ancylostoma spp., Toxocara spp. e Trichuris vulpis, cursam com uma série de sinais clínicos como diarreias, sangramento nasal, epistaxe, vômitos, quadros de anemia e intensa eosinofilia (Paludo et al., 2007; Márquez-Navarro et al., 2012; Ngui et al., 2014) em contaminações isoladas. Casos de contaminação múltipla, estimulado por amostras positivas para vários agentes zoonóticos, conforme evidenciado na Tabela 2, podem potencializar esses efeitos em virtude da ação conjunta dos parasitos, configurando o bairro Simões Lopes como o de maior risco de prevalência para amostras com múltipla infestação $(29,17 \%)$, seguido do bairro Bom Jesus $28,42 \%$. Estes dados divergem de Scaini et al. (2003) que em estudo avaliando a contaminação parasitária de 
237 análises fecais de cães do balneário Cassino (Rio Grande-RS), demonstrou a presença de associações de parasitos em uma única amostra. Porém, associa-se mais intimamente aos dados de
Lopes et al. (2014) que demonstrou, contaminação múltipla por formas parasitárias em $36,62 \%$ de amostras de fezes colhidas na praça pública do município de Pelotas.

Tabela 2. Contaminações mistas de parasitos por amostra de fezes obtidas em vias públicas na cidade de Pelotas-RS entre maio a outubro de 2016.

\begin{tabular}{lccc}
\hline & Bairro & $\mathbf{N}^{\mathbf{0}}$ amostras & Contaminações mistas \\
\hline Simões & 72 & $21(29,17 \%)$ \\
Barro Duro & 122 & $25(20,49 \%)$ \\
Bom Jesus & 95 & $27(28,42 \%)$ \\
Navegantes & Total & 121 & $26(21,49 \%)$ \\
\hline \multicolumn{2}{r}{} & $\mathbf{4 1 0}$ & $\mathbf{1 0 8}$ \\
\hline
\end{tabular}

Os dados obtidos neste trabalho demonstram semelhança ao estudo de prevalência de Beiromvand et al. (2012), com cães errantes e domiciliados em uma área rural do Iran, expondo dados de $42 \%$ de contaminação múltipla. Esta verossimilhança dos dados pode ser reiterada pelas condições medidas pelo índice de desenvolvimento humano (IDH) dos locais de estudo destes dois trabalhos, pois o Brasil e o Iran apresentam alto IDH porém sob a ótica de nutrição, qualidade de saúde, educação e alfabetização são qualificados como países em desenvolvimento (ONU, 2014). O alto potencial de contaminação parasitária de fezes contaminadas no ambiente, não deriva somente dos cães não domiciliados estimados em um número de 13.000, que circulam livremente pelas vias públicas, mas também em cães comunitários e domiciliados que possuem tutores ou guardiões, porém, tem acesso a rua, sendo estes estimados em um número de 45.500 no município de Pelotas segundo levantamento realizado pela Gerência de Vigilância Ambiental do município de Pelotas no ano de 2013 (Lima, 2013). Outro fator que impacta e favorece a mantença de pontos de contaminação de parasitos zoonóticos no município, é a falta de adoção e realização de algumas medidas profiláticas, pois segundo Domingues et al. (2015) em questionários realizado com tutores de animais de estimação abrangendo cães e gatos no município de Pelotas, 26,7\% do total de 1723 entrevistados permitiam o livre acesso de seus animais a rua e somente $25,7 \%$ do total recolhia as fezes dos mesmos e descartava em local apropriado, mesmo que $72,3 \%$ tivesse alegado que realizaram utilização de produto anti-helmíntico em seus animais nos últimos seis meses. Sanchez-Ortiz. (2011) em questionário realizado junto a população da cidade de Ilha Solteira-MS, relevou o risco de transmissão de zoonoses pelo fato de fezes de animais não tratados ficarem constantemente dispostas em locais de trânsito de pessoas e inclusive de crianças.

\section{Conclusão}

A contaminação ambiental nos bairros Bom Jesus, Navegantes, Simões Lopes e Barro Duro do município de Pelotas-RS pelos parasitos zoonóticos Ancylostoma spp., Toxocara spp. e Trichuris vulpis causadores das enfermidades larva migrans cutânea, larva migrans visceral e tricuríase é acentuada em glebas representativas das amostragens colhidas, de forma isolada ou mista. Esse resultado, traz à tona a grande importância da análise parasitológica, controle parasitário e adoção de medidas de profilaxia, extremamente relevantes para os cães não domiciliados, domiciliados e comunitários bem como, para o ser humano, sendo de grande valor científico e social a continuidade de estudos que abranjam o âmbito da saúde pública.

\section{Conflito de interesses}

Os autores afirmam não existir conflito de interesses.

\section{Comitê de Ética}

O projeto foi aprovado pelo Comitê de Ética em Pesquisa da Universidade Federal de Pelotas, CAAE 60713416.0.0000.5317.

\section{Agradecimentos}

Ao programa de residência multiprofissional em Medicina Veterinária área de concentração de Doenças e Zoonoses Parasitárias da Universidade Federal de Pelotas pelo aporte científico e a 
prefeitura Municipal de Pelotas-RS pelo suporte logístico e interesse na melhoria da saúde pública.

\section{Referências}

Anaya, A.M.D.; Medellín, M.O.P.; Forero, G.J.C. Nematodes with zoonotic potential in parks of the city of Tunja, Colombia. Salud Publica De Mexico, 57(2): 170-176, 2015.

Becker, A.C.; Rohen, M.; Epe, C.; Schnieder, T. Prevalence of endoparasites in stray and fostered dogs and cats in Northern Germany. Parasitology Research, 111: 849-857, 2012.

Beiromvand, M.; Akhlaghi, L.; Massom, S.H.F.; Meamar, A.R.; Motevalian, A.; Oormazdi, H.; Razmjou, E. Prevalence of zoonotic intestinal parasites in domestic and stray dogs in a rural area of Iran. Preventive Veterinary Medicine, 109(1): 162-167, 2012.

Brilhante, A.F.; Nunes, V.L.B.; Dorval, M.E.C. Presença de Toxocara spp. e ancilostomídeos em áreas de peridomicílios de uma comunidade pesqueira no Centro-Oeste do Brasil. Brazilian Journal of Veterinary Research Animal Science, 50(1): 71-73, 2013.

Dantas-Torres, F.; Otranto, D. Dogs, cats, parasites, and humans in Brazil: opening the black box. Parasites \& Vectors, 7(1): 22, 2014.

Domingues, L.R.; Cesar, J.A.; Fassa, A.G.; Domingues, M.R. Guarda responsável de animais de estimação na área urbana do município de Pelotas, RS, Brasil. Ciência \& Saúde Coletiva, 20(1): 185, 2015.

Franke, D.; Strube, C.; Epe, C.; Welz, C.; Schnieder, T. Larval migration in PERL chambers as an in vitro model for percutaneous infection stimulates feeding in the canine hookworm Ancylostoma caninum. Parasites \& Vectors, 4(7), 2011.

González, C.G.; Galilea, N.M.; Pizarro, K. Larva migrans cutânea autóctona em Chile. A propósito de um caso. Revista Chilena de Pediatria, 86(6): 426-429, 2015.

Jenkins, D.J.; Lievaart, J.J.; Boufana, B.; Lett, W.S.; Bradshaw, H.; Armua-Fernandez, M.T. Echinococcus granulosus and other intestinal helminths: current status of prevalence and management in rural dogs of eastern Australia. Australian Veterinary Journal, 92(8): 292-298, 2014.

Johnson, S.A.M.; Gakuya, D.W.; Mbuthia, P.G.; Mande, J.D.; Maingi, N. Prevalence of gastrointestinal helminths and management practices for dogs in the Greater Accra region of Ghana. Heliyo, 1(1), 2015.

Labruna, M.B., Pena, H.F.J., Souza, S.L.P., Pinter, A., Silva, J.C.R., Ragozo, A.M.A.; Camargo, L.M A.; Gennari, S.M. Prevalência de endoparasitas em cães da área urbana do município de Monte Negro, Rondônia. Arquivos do Instituto Biológico, 73(2): 183193, 2006.

Leite, L.C.; Círio, S.M.; Navarro-Silva, M.A.; Zadorosnei, A.C.B.; Luz, Ê.; Marinoni, L.P.; Lunelli, D. Ocorrência de endoparasitas em amostras de fezes de cães (Canis familiaris) da região metropolitana de Curitiba, Paraná Brasil. Estudos de Biologia, 68: 319-326, 2007.

Lima, J. Prefeitura Municipal de Pelotas-RS. Gerência de Vigilância AmbientalPrefeitura de Pelotas. Prefeitura faz convênio com SOS Animais para castração de cães 2013. Disponível em: $<$ http://www.pelotas.rs.gov.br/noticias/detalh e.php? controle $=\mathrm{MjAxMy0xMi0xMA== \& co}$ dnoticia=35745>.. Acesso em: 13 fev. 2017.

Lopes, T.V.; Fernandes, C.P.M.; Michelon, L.; Hijano, A.; Félix, S.R.; Schons, S.; Nobre, M. O. Parasitas zoonóticos em fezes de cães de praças públicas em municípios da região sul do Rio Grande do Sul, Brasil. Revista Brasileira de Higiene e Sanidade Animal, 8(2): 242-250, 2014.

Ma, G.; Holland, C.V.; Wang, T.; Hofmann, A.; Fasn, C-K.; Maizels, R.M.; Hotez, P.J.; Gasser, R.B. Human toxocariasis. The Lancet Infectious Diseases, 18: 14-24, 2018.

Mahdy, M.A.; Lim, Y.A.; Ngui, R.; Fatimah, M.S.; Choy, S.H.; Yap, N.J.; Surin, J. Prevalence and zoonotic potential of canine hookworms in Malaysia. Parasites \& Vectors, 5(88): 1-7, 2012.

Monteiro, M.F.M.; Ramos, R.A.N.; Calado, A.M.C.; Lima, V.F.S.; Ramos, I.C.N.; Tenório, R.F.L.; Faustino, M.A.G.; Alves, L.C. Gastrointestinal parasites of cats in Brazil: frequency and zoonotic risk. Revista Brasileira de Parasitologia Veterinária, 25(2): 254-257, 2016.

Mota, K.C.P.; Gómez-Hernández, C.; RezendeOliveira, K. Frequência de enteroparasitos em amostras de fezes de cães em um município do pontal do Triângulo mineiro, Minas Gerais, 
Brasil. Revista de Patologia Tropical, 43(2): 219-227, 2014.

Márquez-Navarro, A.; García-Bracamontes, G.; Álvarez-Fernández, B.E.; Ávila-Caballero, L. P.; Santos-Aranda, I.; Díaz-Chiguer, D.L.; Nogueda-Torres, B. Trichuris vulpis (Froelich, 1789) infection in a child: a case report. Korean Journal Parasitologic, 50(1): 69-72, 2012.

Ngui, R.; Lim, Y.A.; Ismail, W.H.W.; Lim, K.N.; Mahmud, R. Case report: zoonotic Ancylostoma ceylanicum infection detected by endoscopy. American Journal of Tropical Medicine, 91(1): 86-88, 2014.

O'Lorcain, P. Epidemiology of Toxocara spp. in stray dogs and cats in Dublin, Ireland. Journal of Helminthology, 68(4), 331-336, 1994

ONU. Nações Unidas do Brasil. Human development report 2015. Disponível em: $<\mathrm{http} / / /$ report.hdr.undp.org/>. Acesso em: 10 jan. 2017.

Paludo, M.L.; Falavigna, D.L.; Elefant, G.R.; Gomes, M.L.; Baggio, M.L.; Amadei, L.B.; Falavigna-Guilherme, A.L. Frequency of Toxocara infection in children attended by the health public service of Maringá, south brazil. Revista do Instituto de Medicina Tropical, 49(6): 343-348, 2007.

Paiva, A.B.; Souza, F.S.; Lisbôa, R.D.S. Ocorrência de parasitos com potencial zoonótico em áreas de feiras públicas da cidade de Manaus, AM. Revista Brasileira de Higiene e Sanidade Animal, 8(4): 234-242, 2014.

Park, K.; Park, H.; Hwang, H.; Ryu, J.; Lee, K.; Jang, K. 2018. Case report: space-occupying lesion in the liver related to preprandial hunger and hepatic visceral larva migrans. The American Journal of Tropical Medicine and Hygiene, 99(6): 1602-1605, 2018
Pastore, R.; Vitali, L.H.; Weirich, J.; Tojal, A.C.; Macedo, V.D.O.; Prata, A. Hidatidose policística: Relato de dois casos procedentes de Sena Madureira, Acre, na Amazônia brasileira. Revista da Sociedade Brasileira de Medicina Tropical, 36(1): 97-101, 2003.

Peacock, J.; Chur-Hansen, A.; Winefield, H. Mental health implications of human attachment to companion animals. Journal of Clinical Psychology, 68(3): 292-303, 2012.

Sanchez-Ortiz, I.A.S.; Leite, M.A. Fatores de risco da transmissão de zoonoses por costumes da populacão de Ilha Solteira, Brasil (Report). Revista de Salud Publica, 13(3): 504, 2011.

Savilla, T.M.; Joy, J.E.; May, J.D.; Somerville, C.C. Prevalence of dog intestinal nematode parasites in south central West Virginia, USA. Veterinary Parasitology, 178(1): 115-120, 2011.

Scaini, C.J.; Toledo, R.N.; Lovatel, R.; Dionello, M.A.; Gatti, F.A.; Susin, L.; Signorini, V.R. M. Contaminação ambiental por ovos e larvas de helmintos em fezes de cães na área central do Balneário Cassino, Rio Grande do Sul. Revista da Sociedade Brasileira de Medicina Tropical, 36 (5): 617-619, 2003.

Soares, S.; Liz, C.F.D.; Cardoso, A.L.; Machado, Â.; Cunha, J.; Machado, L. Larva Migrans Cutânea: apresentação típica de dois casos clínicos. Nascer e Crescer, 27: 46-49, 2018.

Thomas, D; Jeyathilakan, N. Detection of Toxocara eggs in contaminated soil from various public places of Chennai city and detailed correlation with literature. Journal of Parasitic Diseases, 38(2): 174-80, 2014.

Traversa, D. Are we paying too much attention to cardiopulmonary nematodes and neglecting old fashioned worms like Trichuris vulpis? Parasites \& Vectors, 4(32): 2-11, 2011.

Willis, H.H. A simple levitation method for detection of hookworm ova. Medical Journal of Australia, 8: 375-376, 1921. 